\title{
Fondo Constitucional de Financiamiento del Nordeste del Brasil: efectos diferenciados sobre el crecimiento económico de los municipios
}

\author{
Ricardo Brito Soares, Fabricio Carneiro Linhares, \\ Marcos Falcão Gonçalves y Luiz Fernando Gonçalves Viana
} Constitucional de Financiamiento del Nordeste (FNE) en el crecimiento económico de los municipios de esa región en la década de 2000. Para ello se utiliza un marco empírico basado en modelos de crecimiento que permiten la formación de clubes de convergencia según el nivel de desarrollo inicial del municipio. Los resultados corroboran la estrategia empírica y revelan la existencia de cuatro grupos de municipios donde los flujos de inversión mediante el FNE afectan al crecimiento de manera diferente. En general, el FNE produce efectos positivos y significativos en la mayoría de los municipios del Nordeste, con excepción de aquellos cuyo producto interno bruto (PIB) per cápita era muy bajo o muy alto a comienzos de la década, donde sus efectos no resultaron significativos.

PALABRAS CLAVE

CLASIFICACIÓN JEL
Crecimiento económico, desarrollo regional, gobierno municipal, financiación del desarrollo, fondos, evaluación, modelos econométricos, Brasil

O21, O40, R11

Ricardo Brito Soares es profesor adjunto del curso de posgrado en economía (CAEN) de la Universidad Federal de Ceará (UFC), Brasil. ricardosoares@caen.ufc.br

Fabrício Carneiro Linhares es profesor adjunto del curso de posgrado en economía (CAEN) de la Universidad Federal de Ceará (UFc), Brasil. flinhares@caen.ufc.br

Marcos Falcão Gonçalves es investigador en el Escritório Técnico de Estudos Econômicos do Nordeste (Etene) del Banco do Nordeste del Brasil (влв) y profesor de la Facultad Cearense, Brasil. marcosfalcaogoncalves@gmail.com

Luiz Fernando Gonçalves Viana es investigador en el Escritório Técnico de Estudos Econômicos do Nordeste (etene) del Banco do Nordeste del Brasil (вNB) y profesor de la Faculdade de Fortaleza (FaFor) y de la Faculdade de Ensino e Cultura do Ceará (FAECE), Brasil. luizfernandogv@bnb.gov.br 


\section{I}

\section{Introducción}

El Fondo Constitucional de Financiamiento del Nordeste (FNE), creado a partir de la Constitución de 1988, tiene como objetivo desarrollar y perfeccionar el sistema productivo de esa zona para reducir las desigualdades históricas entre las regiones. La principal herramienta del fondo consiste en la expansión de la oferta de crédito subsidiado en diversos programas de financiamiento, que comprenden empresas de diversos sectores y tamaños. Este programa de crédito ha evolucionado a lo largo de la décadas de 1990 y de 2000, hasta convertirse en uno de los principales instrumentos de estímulo a la iniciativa empresarial regional y al desarrollo productivo.

La participación del FNE en el total de financiamientos a largo plazo en el Nordeste llegó al 64\% entre 2002 y 2009 , mientras que las inversiones programadas para los años siguientes superaban los 10 mil millones de reales al año ${ }^{1}$. A medida que los recursos del fondo público aumentan y se consolidan como instrumento de política regional, se espera que — como contrapartida social—la capacidad de generar riqueza en las regiones menos desarrolladas se incremente en la misma proporción. $\mathrm{O}$, de manera más pragmática, es de esperar que la inyección de un mayor volumen de este tipo de crédito subsidiado en las economías locales pueda fomentar su crecimiento económico.

Esta posibilidad es objeto de exhaustivos análisis en el contexto de la Unión Europea, donde mediante los llamados fondos estructurales se procura acelerar la dinámica de crecimiento de los países y regiones menos desarrollados que forman parte de ese mercado común ${ }^{2}$. En el caso del Brasil, los estudios dedicados al análisis empírico de la contribución de los fondos regionales al crecimiento económico son escasos. Además de los análisis de impacto en los que se utiliza la matriz de insumo-producto (Mesquita, 1996; Rodrigues, 1998), se destacan solo dos artículos: los de Oliveira y Domingues (2005) y Resende (2012a) ${ }^{3}$. Mientras que en el primero se analizan los efectos del Fondo Constitucional de Financiamiento del Centro-Oeste (FCO) y del Fondo Constitucional de Financiamiento del Norte (FNO) en el

\footnotetext{
1 Véase Banco do Nordeste (2011).

2 Véase una revisión de la literatura en Dall'Erba, Guillain y Gallo (2011).

3 Véanse análisis de impacto del FNE en el nivel microeconómico de las empresas en Silva, Resende y Silveira Neto (2009); Soares, Sousa y Pereira Neto (2009) y Resende (2012a).
}

diferencial de crecimiento de los municipios beneficiarios, en el segundo se examinan los efectos del FNE al mismo nivel de agregación macroeconómica. Un elemento común a estos artículos es la utilización de modelos econométricos tradicionales, con los que se investigan las perspectivas de convergencia entre regiones, según el enfoque de Barro y Sala-i-Martin (1992) ${ }^{4}$. Los resultados de ambos trabajos indicaron la ausencia de un efecto medio de las variables relacionadas con los fondos.

Sin embargo, al evaluar los efectos de los fondos públicos en el sector municipal, no se puede ignorar que su importancia y sus repercusiones pueden variar en municipios con diferentes niveles de desarrollo inicial. En las macrorregiones con relativo atraso económico (Norte, Nordeste y Centro-Oeste), la heterogeneidad de los municipios es evidente y está institucionalizada en la propia Política Nacional de Desarrollo Regional (PNDR) (Ministerio de Integración Nacional, 2005). En esta se distinguen cuatro tipos de municipios (de altos ingresos, de bajos ingresos, dinámicos y estancados) a partir de la combinación de las variables ingreso domiciliario medio y crecimiento del PIB per cápita, y se proporcionan las pautas para formular líneas de acción específicas adecuadas a cada tipo municipal. En el ámbito de los fondos regionales, además de los planes de distribución de recursos para cada conjunto de municipios tipificados, el Ministerio de Integración Nacional exige a los administradores la presentación de informes de seguimiento y resultados de acuerdo con esta clasificación intrarregional ${ }^{5}$.

Esta preocupación por la heterogeneidad en las dinámicas de crecimiento se incorporó a una gama de modelos no lineales, que permiten la formación de clubes de convergencia, según el enfoque propuesto por Durlauf y Johnson (1995). Con esos modelos es posible probar y estimar los efectos diferenciados de los cofactores que afectan al crecimiento económico. La formación de clubes de convergencia en el sector municipal, por ejemplo, muestra los municipios que tienen posibilidades

\footnotetext{
${ }^{4}$ Cabe destacar que en cada artículo se agregaron a los modelos de convergencia especificidades de los respectivos estudios de caso, como los efectos espaciales en Oliveira y Domingues (2005) y la estimación en dos etapas en Resende (2012a).

5 Banco do Nordeste do Brasil (BNB) en el caso del FNE; Banco da Amazônia (BASA) en el caso del FNO, y Banco del Brasil (BB) en el caso del FCO.
} 
de convergencia condicional parecidas entre sí, y diferentes entre los grupos (clubes). Cabe señalar que la identificación y formación de clubes en estos modelos no se realizan de manera $a d h o c$, sino que es el resultado de la estructura del modelo y de los datos. En otras palabras, es la estimación de las dinámicas de crecimiento diferenciadas la que define las tipologías regionales.

La identificación de diferentes patrones de crecimiento en forma endógena ya figura en algunos estudios acerca del Brasil. Sobre la base de modelos de paneles dinámicos, Trompieri Neto, Castelar y Linhares (2008) fueron pioneros en analizar el proceso de convergencia de los PIB per cápita estaduales con la posibilidad de formación de clubes. Utilizando datos de 1985-2007, los autores sugieren la existencia de dos clubes de convergencia de la tasa de crecimiento. En forma análoga, Cabral (2008) también identificó la formación de dos clubes de convergencia en el Brasil empleando la metodología propuesta por Phillips y Sul (2007). De acuerdo con Penna (2011), que aborda el tema con diferentes metodologías, la formación de clubes de convergencia de los estados brasileños es estadísticamente significativa, tanto en modelos de datos de panel como en modelos de datos transversales. En otro estudio, en el que se emplean una técnica econométrica y una base de datos similares a las adoptadas en este trabajo, Oliveira y otros (2011) encuentran pruebas que sugieren la existencia de varios clubes de convergencia basados en la dinámica de los PIB per cápita municipales.

En ese contexto, mediante el presente estudio se procura determinar si los efectos del FNE en el crecimiento de los municipios también pueden variar de acuerdo con su grado de desarrollo inicial. Con ese fin se utiliza el algoritmo de Hansen (2000) para un modelo de crecimiento que incluye como variable de interés el volumen de recursos de los programas del FNE destinados a los municipios. De ese modo, el análisis de los efectos del FNE y su heterogeneidad en el crecimiento económico de los municipios nordestinos realizado en este trabajo constituye un aporte a la literatura sobre el tema.

La existencia de efectos diferenciados no solo mejora las propiedades estadísticas de los modelos de crecimiento estimados, sino que - sobre todo- evidencia la heterogeneidad intrarregional del Nordeste de acuerdo con la Política Nacional de Desarrollo Regional (PNDR). No obstante, es posible y probable que las clasificaciones ad hoc definidas en dicha política y aquellas tomadas de los modelos estimados no coincidan plenamente. En ese caso, además de las consideraciones conceptuales, la dirección de las acciones y los recursos mediante la PNDR pueden producir ineficiencias reales.

Este trabajo se divide en cinco secciones, además de la Introducción. Mientras que en la segunda sección se examina la relación entre el crédito y el crecimiento económico, en la tercera se describen el FNE y su distribución en los municipios del Nordeste. En la cuarta sección se presentan los datos y la metodología utilizados para la estimación del modelo de impacto del FNE. En la quinta sección se exponen y analizan los resultados encontrados y en la sexta, a modo de conclusión, se realizan advertencias y consideraciones inductivas sobre el tema.

\section{II \\ Relación entre el crédito y el crecimiento económico}

Joseph Schumpeter fue uno de los pioneros en la defensa de los medios financieros como impulsores del desarrollo económico. De acuerdo con su concepción, el sistema financiero ofrece un conjunto de servicios que facilitan la canalización del ahorro de la sociedad hacia actividades productivas e innovadoras, que constituyen las piezas claves del crecimiento económico.

Los efectos de los medios financieros en el crecimiento del producto (o de los ingresos) se formalizaron por primera vez en los trabajos de
Goldsmith (1969); McKinnon (1973), y Shaw (1973). Mientras que Goldsmith (1969) hace hincapié en que el desarrollo de la intermediación financiera aumenta la eficiencia del proceso de asignación de inversiones en la economía, McKinnon (1973) y Shaw (1973) subrayan que ese desarrollo puede apalancar la tasa de ahorro de la economía y, por consiguiente, su nivel de inversión. Independientemente de sus perspectivas teóricas, estos autores concluyen que la cantidad y la calidad de los servicios del sector financiero son en 
parte responsables de las diferencias en las tasas de crecimiento de los países.

De acuerdo con De Gregorio y Guidotti (1995) y Levine (2004), este efecto en la tasa de actividad de la economía puede apreciarse en forma sencilla mediante los modelos tradicionales de crecimiento económico, donde la trayectoria de la expansión del producto per cápita hacia el estado estacionario de equilibrio está determinada por la tasa de ahorro y la productividad marginal del capital.

Desde el punto de vista de Goldsmith (1969), tal vez el más difundido, la amplitud de los diferentes servicios en el mercado financiero - como captación de recursos, oferta de crédito, procesamiento de datos de mercado y gestión de riesgos- supone un mejoramiento del proceso de asignación de recursos para estimular el aumento de la productividad. Por ejemplo, Boyd y Prescott (1986) afirman que, sin el sector financiero intermediario, el inversionista tendría que incurrir en altos costos para identificar las oportunidades de inversión y evaluar los riesgos y esto restringiría la dinámica de la asignación de recursos. Algunos autores, como Greenwood y Jovanovic (1990); Bencivenga y Smith (1991); Levine (1992), y King y Levine (1993) muestran, mediante modelos de crecimiento endógeno, que el papel analítico e investigativo del intermediario financiero ayuda a dirigir los recursos a los proyectos con mejores posibilidades de presentar avances productivos e innovación, es decir, a los que ofrecen mayor rendimiento.

Desde esa perspectiva, que concuerda con la schumpeteriana, la oferta de crédito de las instituciones financieras, basada en el asesoramiento de sus analistas con respecto a los indicadores de actividad económica y riesgo, puede acelerar el crecimiento gracias a sus efectos positivos en la productividad del capital. Según las estimaciones de Bayoumi y Melander (2008), una reducción del $2,5 \%$ de la oferta general de crédito de una economía se traduce en una contracción del PIB de alrededor del 1,5\%. Ese efecto esperado del crédito en la economía es incluso citado por Keynes (1943) en su obra clásica Teoría general de la ocupación, el interés y el dinero, en la que afirma que:

"El crédito es de fundamental importancia para el desarrollo económico, no solo por su impacto directo en la acumulación de capital y sus reflejos en la distribución de ingresos y la actividad innovadora. El crédito tiene también gran importancia en la formación de expectativas, de forma que, si está bien empleado y se garantizan los buenos resultados de las inversiones, produce un aumento del optimismo en la economía".
En concordancia con McKinnon (1973) y Shaw (1973), un sistema financiero operacional es esencial para movilizar el ahorro y reducir las restricciones financieras externas que dificultan la expansión de empresas e industrias. $\mathrm{Al}$ atender diferentes exigencias en cuanto a la formación de una cartera adecuada, la diversidad de los servicios financieros puede constituir un estímulo para los ahorristas. De acuerdo con Jappelli y Pagano (1994), el sector financiero cumple el papel de aliviar las restricciones impuestas sobre los prestatarios en vista de las perspectivas de ingresos futuros, incrementando así la tasa de ahorro agregada. Siguiendo el razonamiento de Bourguignon (2002), las incertidumbres e imperfecciones de los mercados de crédito hacen que el rendimiento del capital dependa principalmente de la riqueza inicial de los individuos, lo que penaliza en gran medida la capacidad de inversión de la sociedad.

En resumen, la oferta de crédito —o, de modo más general, el desarrollo de los medios financieros-influye en el crecimiento económico, ya sea acrecentando la eficacia de la orientación de los recursos hacia las actividades más productivas (con mayores posibilidades de aumento de la productividad) o incrementando la tasa de ahorro y, en consecuencia, la inversión. Como se teoriza en el modelo propuesto por Aghion, Howitt y Mayer-Foulkes (2004), las tasas de crecimiento del producto de las economías con un nivel de desarrollo financiero suficientemente alto convergerán hacia la tasa de crecimiento establecida por la frontera tecnológica mundial, mientras que las tasas de crecimiento del producto de todas las demás economías serán estrictamente menores a largo plazo.

Desde el punto de vista empírico, la relación entre la disponibilidad y la utilización del crédito y el crecimiento económico varía según el estudio de caso y el método de análisis elegido. En general, se observa una correlación de causalidad positiva entre estas variables. En el Brasil, entre otros trabajos que evidencian esta causalidad, se pueden citar Matos (2002); Marques Jr. y Porto Jr. (2004); Chinelatto Neto (2007), y Rocha y Nakane (2007).

Sin embargo, los efectos del crédito subsidiado con fondos públicos en el crecimiento y en la disminución de las desigualdades regionales constituyen un tema menos explorado en la literatura. Si bien el crédito subsidiado como instrumento de política regional no es algo nuevo, solo a partir de la formación de mercados únicos y del auge de los programas de crédito para las clases sociales más bajas en los diversos sectores económicos en los años noventa, el interés en dicho crédito se ha renovado. Por ejemplo, mediante la política de cohesión de la Unión 
Europea - que se basa en la transferencia de fondos estructurales para financiar programas de concesión de crédito- se procura disminuir las desigualdades existentes entre los países miembros apoyando la formación de estructuras productivas y fomentando los mercados locales.
Los efectos de los fondos estructurales europeos en el crecimiento económico siguen siendo una cuestión abierta. Dall'Erba y De Groot (2006) analizaron 11 estudios empíricos realizados durante la década de 2000 y encontraron tanto efectos positivos como insignificantes $\mathrm{o}$ incluso negativos.

\section{III}

\section{El Fondo Constitucional de Financiamiento del Nordeste y los estudios de sus efectos en el crecimiento}

En el Brasil, los fondos constitucionales regionales (FNE, FCO, FNO) se forman a partir de la recaudación anual del $3 \%$ del impuesto sobre los productos industrializados (IPI) y del impuesto a la renta. Su objetivo es mejorar la capacidad de crecimiento de las macrorregiones menos desarrolladas (Nordeste, Centro-Oeste y Norte, respectivamente) para disminuir las desigualdades regionales en el país.

Administrado por el Banco do Nordeste do Brasil (BNB) desde su creación en 1988, el FNE abarca 1.990 municipios situados en todos los estados nordestinos y en el norte de los estados de Minas Gerais y Espírito Santo, incluidos en el área de actuación de la Superintendencia para el Desarrollo del Nordeste (SUDENE) (Brasil, 1999). El financiamiento de las inversiones productivas se realiza mediante programas de préstamos, cuyo principal atractivo son las tasas de interés subsidiadas, que están muy por debajo de las tasas del mercado ${ }^{6}$. A fin de estimular la demanda, además de las disponibilidades de las agencias bancarias, se designan agentes de desarrollo para analizar y orientar las oportunidades locales de negocios en todos los sectores y de todos los tamaños ${ }^{7}$.

Como se puede apreciar en el gráfico 1, el volumen de recursos aplicados ha ido aumentando a lo largo de la existencia del fondo. En particular se observa un incremento considerable a partir de 2002, que obedece tanto al mayor crecimiento de la economía como a

\footnotetext{
${ }^{6}$ Este diferencial de interés es aún mayor para las inversiones destinadas a las regiones menos desarrolladas, como la del semiárido, y a las empresas más pequeñas.

7 Véase un análisis retrospectivo del FNE en Ministerio de Integración Nacional (2010).
}

los estímulos del gobierno tendientes a ampliar la disponibilidad de crédito para la región Nordeste.

Al examinar la distribución sectorial de los recursos en el período 2002 - 2008, se verifica que el FNE financió emprendimientos en todos los sectores económicos. Cabe destacar que el $46,5 \%$ del financiamiento se destinó al sector rural, debido a la propia dinámica de la economía nordestina, tradicionalmente caracterizada por la producción agropecuaria. En segundo lugar figuran el sector industrial y el turismo con el $22,4 \%$ de los recursos invertidos, seguidos por el sector comercio y servicios con el 15,5\% (véase el gráfico 2).

Con respecto al tamaño de los beneficiarios, el valor más representativo corresponde a las grandes empresas, que recibieron el $54,2 \%$ de los recursos en el período analizado en el estudio, seguidas por las mini y pequeñas empresas $(32,6 \%)$ y luego por las medianas $(13,2 \%)$ (véase el gráfico 3 ).

En el mapa 1 se muestra la suma de los recursos distribuidos a cada municipio por intervalos de valores definidos para cada quintil en ese mismo período (en reales de 2007) ${ }^{8}$. Si bien todos los municipios fueron beneficiados, el volumen de recursos recibidos varía considerablemente de uno a otro, de 15.900 reales en Santa Cecília-PB a 1.300 millones de reales en Fortaleza-CE ${ }^{9}$

\footnotetext{
${ }^{8}$ Valores deflacionados por el índice general de precios-disponibilidades internas (IGP-DI). El total de recursos en el período fue de aproximadamente 24.900 millones de reales.

9 Cabe destacar también que, según la clasificación de la PNDR, un $15,38 \%$ de los recursos se destinó a los municipios de bajos ingresos, el $31,12 \%$ a los estancados, el $27,72 \%$ a los dinámicos y el $25,78 \%$ a los de altos ingresos. Asimismo, con respecto a la distribución regional cabe notar que solo el 34,76\% de los recursos se destinó a municipios de la región del semiárido del Nordeste.
} 
GRÁFICO

Contrataciones anuales del Fondo Constitucional de Financiamiento del Nordeste, 1994-2008

(En millones de reales de 2008)

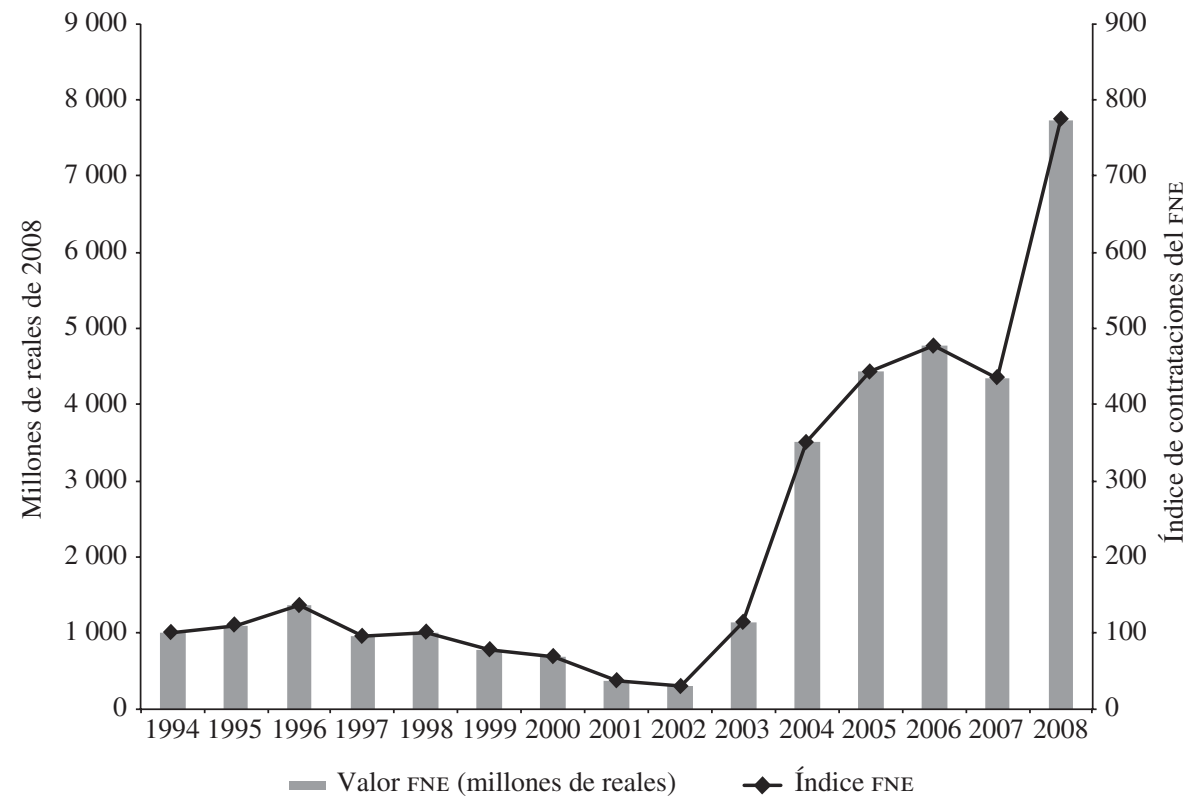

Fuente: Ministerio de Integración Nacional, 20 Anos de Fundos Constitucionais de Financiamento (FCO-FNE-FNO), Brasilia, 2010.

GRÁFICO 2

Contrataciones del Fondo Constitucional de Financiamiento del Nordeste según el sector, 2000-2008

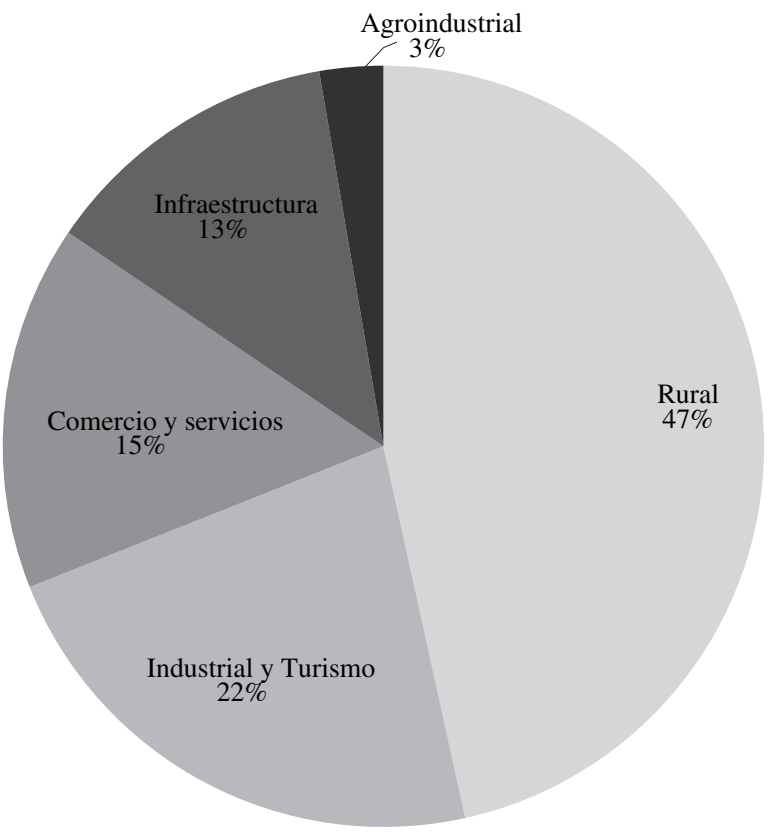

Fuente: elaboración propia, sobre la base de datos del Banco do Nordeste do Brasil. 
GRÁFICO 3

Contrataciones del Fondo Constitucional de Financiamiento del Nordeste según el tamaño de los beneficiarios, 2000-2008

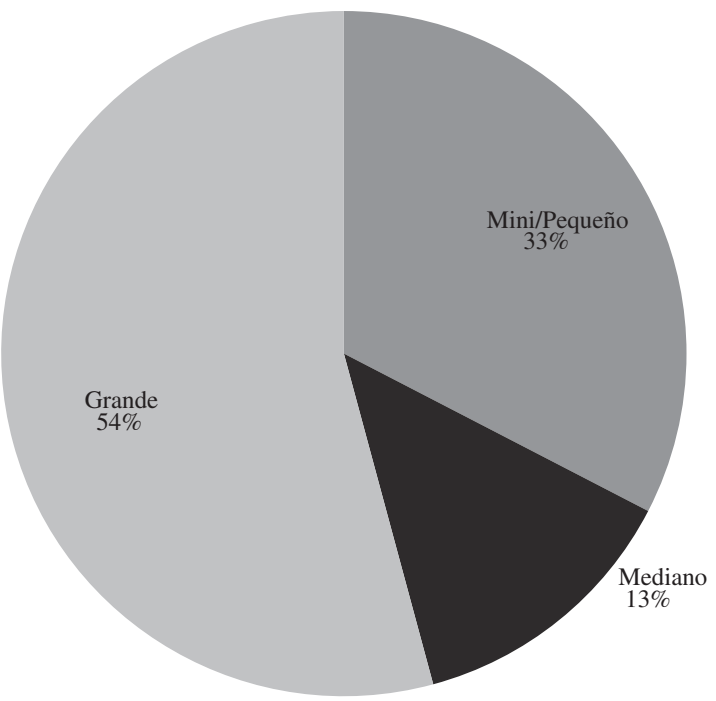

Fuente: elaboración propia, sobre la base de datos del Banco do Nordeste do Brasil.

MAPA 1

Aplicaciones acumuladas del Fondo Constitucional de Financiamiento del Nordeste por municipio, 2002-2008

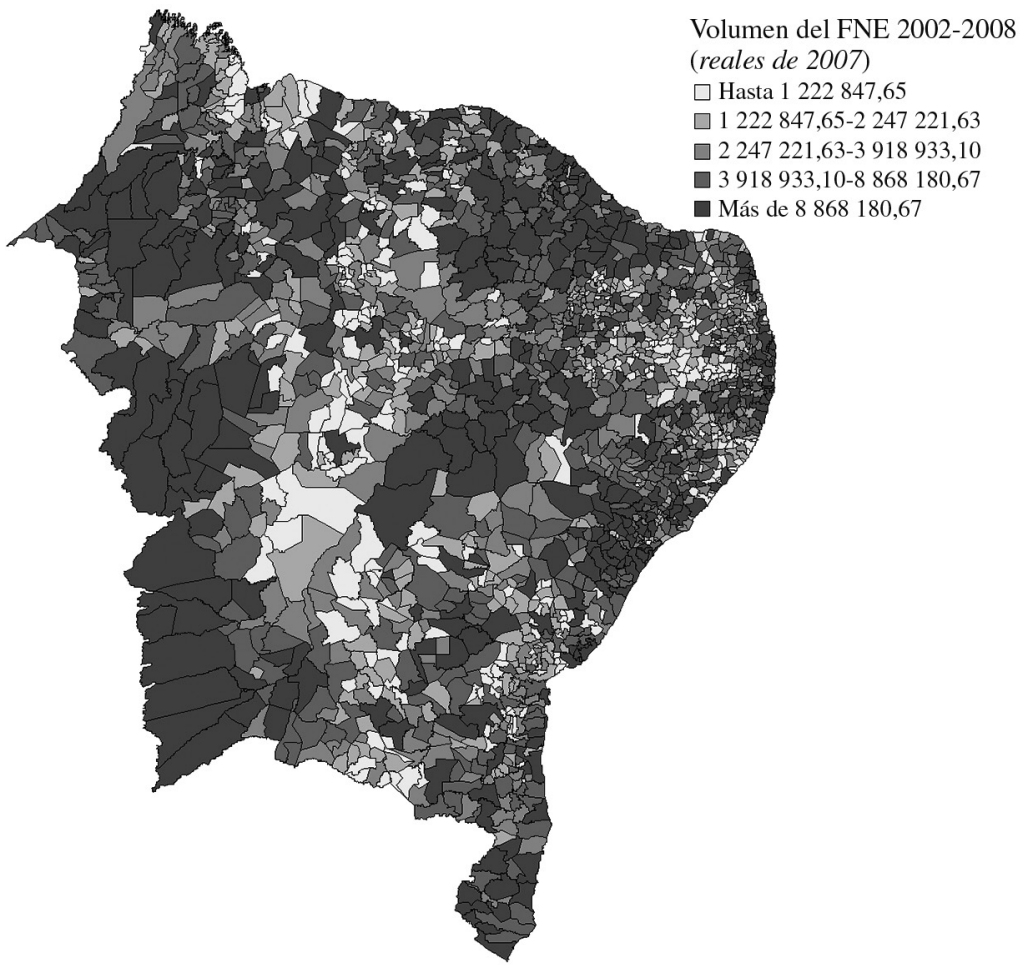

Fuente: elaboración propia, sobre la base de datos del Banco do Nordeste do Brasil. 
en promedio por año. La cuestión de fondo que se plantea a partir de esta constatación es determinar si los municipios que reciben proporcionalmente más recursos, de hecho están creciendo más por efecto del financiamiento.

Los análisis de la eficacia de los fondos constitucionales todavía son escasos. Oliveira y Domingues (2005) procuran determinar si los recursos de los fondos constitucionales del Centro-Oeste y del Norte han contribuido a reducir las diferencias entre las tasas de crecimiento de los municipios de estas regiones y la media nacional. Para ello utilizan un modelo tradicional de crecimiento, que incluye efectos espaciales que representan externalidades económicas. Los resultados de este estudio no evidenciaron un efecto significativo de los fondos en la reducción de las desigualdades.

Resende (2012a) llegó a conclusiones cualitativas similares con respecto al FNE. El autor evalúa los efectos del FNE en el crecimiento económico de los municipios del área de actuación del Banco del Nordeste del Brasil. Como variable calificativa del FNE, utiliza la razón entre el volumen de recursos del fondo (programas industrial, de servicios y de comercio) y el producto interno bruto (PIB) de los municipios en los períodos 2000-2001 y 2000-2003, y como variable de resultado (impacto macroeconómico) emplea el crecimiento del PIB per cápita en los períodos comprendidos entre 2002 y 2006. Para las variables de control se utilizaron indicadores de educación (media de años de estudio), salud (mortalidad infantil), demografía (densidad poblacional), vivienda (índice de infraestructura) y transporte (costo de transporte medido por distancia lineal hasta São Paulo, además de la variable que indica convergencia (PIB per cápita en 2002). Al controlar el potencial problema de endogeneidad del FNE con un modelo estimado en dos etapas, el autor no encuentra un efecto positivo y estadísticamente significativo del FNE en el crecimiento económico.

Si bien en este artículo se comparte el modelo teórico de crecimiento económico observado en Resende (2012a), su verificación empírica difiere en varios aspectos. En primer lugar, el período de crecimiento del PIB investigado es más amplio (2002-2008) y comprende un lapso de mayor crecimiento económico, que puede haber sido inducido por la propia maduración de las inversiones del $\mathrm{FNE}^{10}$. En segundo lugar, se contabilizan los aportes del FNE a todos los programas y no solo a los del sector industrial. Si bien estos últimos tienen la mayor participación en el valor de los recursos del FNE de manera agregada en el período analizado ${ }^{11}$, puede que esto no ocurra en forma homogénea en el sector municipal. Por ese motivo, es necesario probar también otras dimensiones sectoriales del FNE como variables clave de investigación de impacto.

Por último, es posible que los efectos del FNE se sientan de distinta manera en los diferentes municipios del Nordeste. En otras palabras, la verificación de los efectos puede depender del volumen de recursos recibidos con respecto al tamaño de la economía del municipio o de otro indicador económico de este. La variabilidad o heterogeneidad de los efectos macroeconómicos del FNE dentro del conjunto de municipios del Nordeste constituye una posibilidad plausible, que debe probarse empíricamente ${ }^{12}$. De ser válida, su omisión del modelo econométrico se traduce en estimaciones sesgadas e incoherentes.

En este artículo se procura abordar las preocupaciones anteriores mediante la estimación de un modelo de crecimiento no lineal, que capta las heterogeneidades de los efectos de acuerdo con el nivel de desarrollo inicial de los municipios y cubre un período de gran crecimiento para el Nordeste (2002-2008).

\footnotetext{
${ }^{10}$ La elección del período final de 2008 se debe a la restricción de los datos disponibles sobre el PIB de los municipios en el momento de la realización de este trabajo.

11 Además de los programas industrial, comercial y de servicios, el FNE comprende programas para los sectores rural, agroindustrial y de infraestructura. Históricamente, el sector rural es el que presenta el mayor volumen de contrataciones.

${ }^{12}$ Cabe mencionar que la heterogeneidad en los efectos del FNE entre municipios fue destacada por Resende (2012b) en un estudio para el sector industrial de Ceará.
} 


\section{IV}

\section{Metodología y base de datos}

\section{Modelo econométrico}

Los efectos del FNE en los indicadores macroeconómicos de los municipios de esa región se evalúan con una metodología similar a la empleada en los trabajos en el área de crecimiento económico, cuya principal referencia son las obras de Baumol (1986) y Barro y Sala-i-Martin (1991 y 1992). En esos estudios, la hipótesis de que el PIB per cápita de las economías tiende a igualarse a lo largo del tiempo se analiza con un modelo de regresión, que convierte a la tasa media de crecimiento del PIB per cápita entre el período inicial y final $\left(\dot{y}_{i, T}\right)$ en una función del valor del PIB per cápita en el período inicial $\left(y_{i}, 0\right)$,

$$
\dot{y}_{i, T}=\alpha_{1}+\alpha_{2} \ln \left(y_{i, 0}\right)+\varepsilon_{i},
$$

donde:

$\alpha_{1}$ y $\alpha_{2}$ son parámetros;

$\varepsilon_{i}$ es el término de error; $\mathrm{y}$

$i$ es el índice referente a la unidad de observación (economía).

En caso de verificarse la hipótesis de que $\alpha_{2}$ sea negativa y estadísticamente significativa en el contexto de la ecuación (1), las economías presentan un patrón de crecimiento económico coherente con la teoría de la convergencia absoluta.

En estudios posteriores, el modelo empírico de crecimiento se flexibilizó para controlar posibles diferencias a largo plazo en los niveles de PIB per cápita de las economías. Esa propuesta, conocida como regresiones de Solow aumentadas, se discutió inicialmente en Mankiw, Romer y Weil (1992) y se adoptó en varios trabajos posteriores en el marco de la investigación de la convergencia condicional. El modelo de regresión tendría la siguiente forma:

$$
\dot{y}_{i, T}=\alpha_{1}+\alpha_{2} \ln \left(y_{i, 0}\right)+B^{\prime} X_{i}+\varepsilon_{i},
$$

donde:

$X_{i}=\left[x_{l i} \ldots x_{k i}\right]$ es un vector con $k$ variables condicionantes (de control);

$B^{\prime}=\left[b_{1} b_{2} \ldots b_{\mathrm{k}}\right]$ es el vector de coeficientes de pendiente parciales.

El conjunto de regresores para componer $X_{i}$ es amplio e incluye variables que caracterizan aspectos políticos, económicos y sociales de las economías. Véase una lista detallada de esos posibles regresores en Durlauf y Quah (1999) y Durlauf, Johnson y Temple (2004).

Para evaluar el efecto agregado del FNE en los municipios nordestinos se adoptan como esquema inicial las siguientes modificaciones de los modelos de regresión (1) y (2):

$$
\begin{gathered}
\dot{w}_{i, T}=\beta F_{i}+a_{1}+a_{2} \ln \left(w_{i, 0}\right)+\varepsilon_{i}, \\
\dot{w}_{i, T}=\beta F_{i}+a_{1}+a_{2} \ln \left(w_{i, 0}\right)+A^{\prime} X_{i}+\varepsilon_{i},
\end{gathered}
$$

donde:

$\dot{w}_{i, T}$ es la tasa media de crecimiento del indicador macroeconómico $w$ entre 2002 y $2008, w_{i, 0}$ es su valor en 2002;

$F_{i}$ es una medida que capta la intensidad de la política del FNE en el municipio;

$\beta$ es el parámetro que informa el efecto del FNE en el crecimiento de $w$;

$X_{i}=\left[\begin{array}{llll}x_{3 i} & x_{4 i} & \ldots & x_{k i}\end{array}\right]$ es un vector con $k-2$ variables condicionantes (definidas en la sección siguiente);

$A^{\prime}=\left[\begin{array}{llll}\alpha_{3} & \alpha_{4} & \ldots & \alpha_{k}\end{array}\right]$ es el vector de los demás coeficientes de pendiente parciales;

$\varepsilon_{i}$ es el término de error; $\mathrm{y}$

$i$ es el índice referente al $i$-ésimo municipio.

En las ecuaciones (3) y (4), los efectos del FNE se miden por su capacidad de promover el crecimiento del PIB per cápita. Si $w$ es el PIB per cápita y $F$ es el volumen de financiamientos del FNE per cápita, el parámetro $\beta$ puede interpretarse como una elasticidad crecimiento-FNE: un cambio del $1 \%$ en $F$ produce un cambio de $\beta$ puntos porcentuales en la tasa de crecimiento de $w$. Debido a que los recursos de los financiamientos realizados por el FNE generalmente se utilizan para impulsar la economía local y expandir su estructura productiva, se espera que su aplicación en un municipio se traduzca en un aumento de la tasa de crecimiento del ingreso, el empleo y el salario medio $(\beta>0)$.

Uno de los límites de las ecuaciones (3) y (4) en el análisis de los efectos macroeconómicos del FNE es la suposición de que estos son iguales en todas las unidades de corte transversal. En otras palabras, el parámetro que refleja los efectos del FNE, $\beta$, es el mismo para todas 
las economías municipales. Sin embargo, es posible que los municipios con diferentes niveles de desarrollo y estructuras productivas resulten afectados por el FNE en forma desigual.

Aunque reciente, la idea de que los parámetros en los modelos de crecimiento económico varían entre las economías ya se ha discutido bastante. En los trabajos de Azariadis y Drazen (1990) y de Durlauf y Johnson (1995), por ejemplo, se modifica el modelo de crecimiento tradicional, moderando la suposición de convexidad de la función de producción, y se sugiere la posibilidad de múltiples estados estacionarios para el PIB per cápita. En la trayectoria temporal del ingreso, los efectos de los determinantes del crecimiento serían heterogéneos, dependiendo del conjunto de especificidades de las economías.

Para dar cabida a esa posibilidad en el estudio del FNE, se transforman las ecuaciones (3) y (4) incorporando efectos de umbral, conforme con la propuesta de Hansen (2000):

$$
\begin{gathered}
\dot{w}_{i, T}=\left(\beta_{1} F_{i}+a_{11}+a_{21} \ln \left(w_{i, 0}\right)\right) \cdot I_{1}\left\{y_{i, 0} \leq \gamma_{1}\right\}+ \\
\left(\beta_{2} F_{i}+a_{12}+a_{22} \ln \left(w_{i, 0}\right)\right) \cdot I_{2}\left\{\gamma_{1}<y_{i, 0} \leq \gamma_{2}\right\}+\ldots,(5) \\
\left(\beta_{J} F_{i}+a_{1 J}+a_{2 J} \ln \left(w_{i, 0}\right)\right) \cdot I_{J}\left\{y_{i, 0}>\gamma_{J-1}\right\}+\varepsilon_{i} \\
\dot{w}_{i, T}=B_{1}^{\prime} Z_{i} \cdot I_{1}\left\{y_{i, 0} \leq \gamma_{1}\right\}+B_{2}^{\prime} Z_{i} \cdot I_{2}\left\{\gamma_{1}<y_{i, 0} \leq \gamma_{2}\right\}+\ldots, \\
B_{J-1}^{\prime} Z_{i} \cdot I_{J-1}\left\{\gamma_{J-2}<y_{i, 0} \leq \gamma_{J-1}\right\}+ \\
B_{J}^{\prime} Z_{i} \cdot I_{J}\left\{y_{i, 0}>\gamma_{J-1}\right\}+\varepsilon_{i},
\end{gathered}
$$

donde:

$Z_{i}=\left[F_{i} 1 \ln \left(w_{i}, 0\right) x_{3 i} X_{4 i} \ldots x_{k i}\right]$ es un vector de regresores;

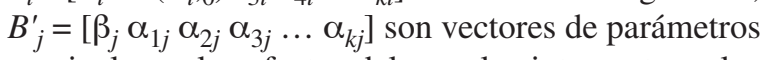
que incluyen los efectos del FNE, los interceptos y los coeficientes de pendiente parciales;

$I_{j}\{\cdot\}$ son funciones indicadoras con $I_{j}\{\Psi\}=1$ en caso de producirse $\Psi$; y

$I_{j}\{\Psi\}=0$ de otra manera;

$y_{i, 0}$ es la variable de umbral que influye en los cambios en los parámetros; valor del PIB per cápita en 2002;

$\gamma_{j}$ son los parámetros de umbral;

$j=1, \ldots, J$ es el índice relativo al régimen o grupo de municipios que comparten los mismos valores de los parámetros $B_{j}$, y $\varepsilon_{i}$ es el término de error.

Estos modelos permiten evaluar los efectos macroeconómicos del FNE en $J$ posibles grupos de municipios, seleccionados de acuerdo con el PIB per cápita municipal en 2002 (variable sustitutiva del nivel de desarrollo de la economía). La heterogeneidad en el efecto del FNE se caracteriza por la variación en los coeficientes $\beta_{j}$ y, por consiguiente, en los vectores $B_{j}$; o sea, en la influencia de todos los determinantes de $\dot{w}_{i, T}$. En caso de invariabilidad de los parámetros, $B_{1}=\ldots=B_{J}$, los modelos de regresión se reducirían a las primeras formas, expresadas por las ecuaciones (3) y (4).

La variación en los efectos del FNE se investiga, por lo tanto, a través de pruebas sobre restricciones en el vector de parámetros $B_{j}$, condicional a las funciones indicadoras $I_{j}\{\cdot\}$. En el proceso de estimación de los modelos, esas pruebas permiten ver la cantidad relevante de grupos en la evaluación de los efectos del FNE y determinan el nivel del PIB per cápita inicial que define a cada grupo. En otras palabras, junto con las estimaciones de los parámetros $\gamma_{j}$ se obtiene estáticamente el valor de $J$.

Para simplificar la explicación de la estimación del modelo y la determinación de $J$ (el número de grupos), se debe considerar la ecuación (6) con solo dos grupos posibles. El grupo 1 estaría compuesto por municipios con un PIB per cápita inicial inferior a $\gamma_{1}$ y el grupo 2 por municipios con un PIB per cápita inicial superior a $\gamma_{1}$.

$\dot{w}_{i, T}=B_{1}^{\prime} Z_{i} \cdot I_{1}\left\{y_{i, 0} \leq \gamma_{1}\right\}+B_{2}^{\prime} Z_{i} \cdot I_{2}\left\{y_{i, 0} \leq \gamma_{1}\right\}+\varepsilon_{i}$.

La ecuación (7) puede reescribirse de la siguiente forma:

$$
\dot{w}_{i, T}=\Phi\left(\gamma_{1}\right)^{\prime} Z_{i} \cdot+\varepsilon_{i}
$$

$\operatorname{con} \Phi\left(\gamma_{1}\right)^{\prime}=\left[B_{1}^{\prime} I_{1}\left\{y_{i, 0} \leq \gamma_{1}\right\} \quad B_{2}^{\prime} I_{2}\left\{y_{i, 0}>\gamma_{1}\right\}\right]$

El procedimiento de estimación de la ecuación (8) sigue la metodología propuesta en Hansen (2000). Sea $P$ el intervalo formado por el mínimo y el máximo de la variable de umbral en la muestra, $P=\left[\min _{i}\left\{y_{i, 0}\right\}\right.$, $\left.\max _{i}\left\{y_{i, 0}\right\}\right]$. Para un $\mu \in(0,1)$, se define $\Gamma$ como la versión discreta del espacio $P$ con $100 . \mu \%$ de sus valores iniciales y finales excluidos simétricamente ${ }^{13}$, formada por $m$ puntos equidistantes. En primer lugar, el vector de parámetros $\Phi$ se estima por mínimos cuadrados para todo $\gamma_{1} \in \Gamma$, formando $m$ valores para la suma del

13 La exclusión de esos valores es una condición necesaria para el buen desempeño en la estimación de los parámetros y la ejecución de las pruebas. 
cuadrado de los residuos $S\left(\gamma_{1}\right)=\sum_{i}\left(\dot{w}_{i, T}-\tilde{\Phi}\left(\gamma_{1}\right)^{\prime} Z_{i}\right)^{2}$. A continuación, se obtienen las estimaciones de $\Phi$ y $\gamma_{1}$ encontrando el valor de $\gamma_{1} \in \Gamma$ que minimiza la función $S\left(\gamma_{1}\right),\left\{\hat{\Phi}, \hat{\gamma}_{1}\right\}=\underset{\gamma_{1} \in \Gamma}{\operatorname{argmin}} S\left(\gamma_{1}\right)$.

Al final de ese procedimiento se obtienen dos conjuntos de coeficientes estimados, $\hat{B}_{1}$ para los municipios con $\left\{y_{i, 0} \leq \hat{\gamma}_{1}\right\}$ y $\hat{B}_{2}$ para los municipios con $\left\{y_{i, 0}>\hat{\gamma}_{1}\right\}$. La prueba de heterogeneidad de los parámetros bajo el efecto de umbral, $B_{1} \neq B_{2}$, que informa si estadísticamente existen dos grupos de municipios distintos (por el contexto del modelo de regresión), utiliza la estadística de la prueba del multiplicador de Lagrange, propuesta por Hansen (1996). Los valores críticos de dicha estadística, robusta a la heterocedasticidad, se determinan mediante un procedimiento de bootstrap. En caso de constatarse que $B_{1} \neq B_{2}$, se concluye que existen dos grupos en los cuales el FNE y las otras variables de control producen efectos distintos en la tasa de crecimiento de $w$.

Al constatar la presencia de dos grupos, el análisis procede con la prueba de la existencia de tres grupos. En ese caso, el vector de parámetros de la ecuación (8) está dado por

$$
\begin{aligned}
& \Phi\left(\gamma_{1}, \gamma_{2}\right)^{\prime}= \\
& {\left[B_{1}^{\prime} I_{1}\left\{y_{i, 0} \leq \gamma_{1}\right\} B_{2}^{\prime} I_{2}\left\{\gamma_{1}<y_{i, 0} \leq \gamma_{2}\right\} B_{3}^{\prime} I_{3}\left\{y_{i, 0}>\gamma_{2}\right\}\right]}
\end{aligned}
$$

o por

$$
\begin{aligned}
& \Phi\left(\gamma_{1}, \gamma_{2}\right)^{\prime}= \\
& {\left[B_{1}^{\prime} I_{1}\left\{y_{i, 0} \leq \gamma_{2}\right\} \quad B_{2}^{\prime} I_{2}\left\{\gamma_{2}<y_{i, 0} \leq \gamma_{1}\right\} B_{3}^{\prime} I_{3}\left\{y_{i, 0}>\gamma_{1}\right\}\right] .}
\end{aligned}
$$

El vector de parámetros $\Phi$ se estima por mínimos cuadrados para todo $\gamma_{2} \in \Gamma$, con $\left|\gamma_{2}-\gamma_{1}\right| \geq \delta>0$, formando varios valores para la suma del cuadrado de los residuos $S\left(\gamma_{1}, \gamma_{2}\right)=\sum_{i}\left(\dot{w}_{i, T}-\tilde{\Phi}\left(\gamma_{1}, \gamma_{2}\right)^{\prime} Z_{i}\right)^{2}$ y, de manera similar, las estimaciones de $\Phi, \gamma_{1}$ y $\gamma_{2}$ se obtienen minimizando la función $S\left(\gamma_{1}, \gamma_{2}\right)$ en el espacio de valores de umbral. Por último, el modelo con tres grupos se confronta con el modelo de dos grupos mediante la prueba del multiplicador de Lagrange. Si el modelo con tres regímenes —o dos parámetros de umbral— resulta más adecuado estadísticamente, se continúa aplicando el mismo procedimiento de estimación y prueba hasta determinar el número de regímenes del modelo.

\section{Datos}

La muestra empleada en este estudio contiene datos sobre el PIB per cápita de los municipios del Nordeste, la cantidad de recursos de los programas del FNE aplicados en esas localidades y las variables (cofactores) que caracterizan e influyen en la composición económica de los municipios, de acuerdo con la propuesta del modelo de crecimiento (4). Todas las variables monetarias están deflacionadas por el índice general de precios-disponibilidades internas sobre la base de $2007^{14}$.

En las ecuaciones de regresión, $\dot{w}_{i, T}$ y $w_{i, 0}$ se refieren entonces a la tasa media de crecimiento del PIB per cápita de cada municipio en el período 2002-2008 y al PIB per cápita de cada municipio en el año 2002. Es importante señalar que el valor del PIB per cápita en 2002 también se emplea como variable de umbral, es decir, la variable sobre la que se formarán (o no) grupos de municipios con patrones de crecimiento similares ${ }^{15}$.

La variable sobre la que se evaluará el efecto de los recursos del FNE en el crecimiento de esos indicadores, $F_{i}$, es la media per cápita anual del total de financiamientos del FNE en el período 2002-2006 ${ }^{16}$. De ese modo, se dejan por lo menos dos años de descanso para captar la maduración de las inversiones en el crecimiento del municipio y se evitan, desde el punto de vista metodológico, los problemas de endogeneidad de la política en las estimaciones del modelo ${ }^{17}$.

Debido a que el FNE está formado por varios programas de financiamiento que se concentran en diferentes aspectos del mercadeo, es posible que el efecto del fondo en un determinado municipio dependa del tipo de municipio y del tipo específico de programa que se desarrolla con mayor intensidad en ese lugar. Los programas como el Programa Nacional de Fortalecimiento de la Agricultura Familiar (PRONAF) y el FNE-Industrial tienen clientes diferenciados cuya actuación y contribución al crecimiento del mercado se realizan por medio de mecanismos distintos. Por ejemplo, es más probable que el primero produzca efectos en la demanda, pues la economía de subsistencia no supondría un avance en la estructura productiva, no obstante que la suma de los pequeños agricultores familiares promueva la formación de cadenas productivas organizadas. Por el contrario, el FNE-Industrial comportaría efectos en la oferta, pues representa en muchos casos la propia expansión de la estructura productiva. Si bien los efectos multiplicadores del FNE-Industrial son acentuados, no se puede dejar de

\footnotetext{
14 Véase Ipeadata [en línea] www.ipeadata.gov.br.

15 Los valores del PIB per cápita relativos a los municipios fueron tomados de Ipeadata.

16 Los valores del FNE relativos a los municipios fueron tomados de ETENE/BNB.

17 Los resultados de los modelos estimados con esa variable con respecto al período 2002-2005 fueron bastante similares.
} 
lado la participación del PRONAF en el acrecentamiento de las economías locales. Por ese motivo, se optó por utilizar el total del FNE para representar la variable de política y no solo los recursos proporcionados por los programas industriales.

Las variables $x_{3 i} x_{4 i} \ldots x_{k i}$, presentadas en la subsección 1 , son otros determinantes del crecimiento económico o variables de control utilizadas en diversos análisis empíricos en esta línea de investigación ${ }^{18}$ Se eligen por su idoneidad para el estudio y su disponibilidad con respecto a todos los municipios en el período 20022008. En consecuencia, se seleccionaron las siguientes variables: el logaritmo de la media de las razones entre gasto corriente y PIB municipal en 2002 (gov), que representa el tamaño del gobierno; el logaritmo de la media de años de estudio de las personas con 25 años o más en $2000(e d u)$, que representa las condiciones

18 Véase la revisión presentada en Tsangarides (2005).

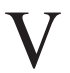

\section{Resultados}

En esta sección se presentan los principales resultados de los modelos lineales (1) y (2), denominados globales, y los modelos con efecto de umbral (3) y (4). El proceso de estimación de esos modelos se basó en la metodología descrita en la sección IV, empleándose además estadísticas de prueba y estimaciones de desviaciones estándar robustas a la heterocedasticidad debido al rechazo de la hipótesis de homocedasticidad de los residuos ${ }^{19}$. A pesar de que se presentan los modelos lineales, estos fueron rechazados por la prueba del multiplicador de Lagrange al realizar la comparación con los modelos con efecto de umbral. Eso indica que estos últimos son estadísticamente más adecuados para la descripción de los datos.

Los resultados completos de los modelos se detallan en el anexo (véanse los cuadros A.2 y A.3), mientras que en los cuadros que aparecen a continuación se presentan solo las estimaciones de los efectos del FNE en la tasa media de crecimiento del PIB per cápita. Los modelos se basan en un número diferente de observaciones (municipios), pues la selección de la muestra se realiza

${ }^{19}$ Se utilizó una versión adaptada de la rutina de Gauss proporcionada por Hansen (2000). de educación y capital humano de los municipios; el logaritmo de la suma de la tasa media de crecimiento de la población de los municipios en el período de 2002 a $2008(n)$; la tasa de crecimiento de la tecnología y la tasa de depreciación $(\delta+d)=5 \%$; la proporción de hogares con agua por cañería (infra), que es una medida de infraestructura; el volumen total de operaciones de crédito $(o p c)$; la distancia del municipio con relación a la capital del respectivo estado (dist), que capta efectos geográficos e indirectos; $y$ una variable binaria que indica si el municipio está situado en una región semiárida (semi). Véase una descripción más detallada de estas variables y sus fuentes en el cuadro A.1 del anexo.

Conviene destacar que la variable relativa a las operaciones de crédito constituye un importante control para la identificación del efecto del FNE en el crecimiento de los municipios. Al controlar los posibles efectos del total de crédito dirigido a los municipios, el efecto del FNE se distingue de otras posibilidades de financiación bancaria.

en función de la disponibilidad de datos relativos a todas las variables en la ecuación de regresión estimada.

Hay que recordar que la variable de umbral utilizada en el análisis es el logaritmo natural del PIB per cápita inicial, que en este estudio corresponde al año 2002. El uso del PIB per cápita inicial como dicha variable es muy común en trabajos similares y, en el contexto de los modelos empíricos, sirve como variable sustitutiva para dividir los municipios en grupos de acuerdo con el estado de desarrollo de sus economías al inicio del proceso de crecimiento analizado. Así, en el caso de los modelos (5) y (6), se podría interpretar que los efectos del FNE se separan en grupos de municipios con niveles de desarrollo distintos.

En los cuadros 1 y 2 se indica el tamaño de la muestra (número de municipios) y se presentan las estimaciones de los efectos del FNE en la tasa media de crecimiento del PIB per cápita correspondientes al modelo global y al modelo con efecto de umbral, respectivamente, según se utilicen o no las variables de control $X_{i}$. Al incluir los controles, la muestra pasa de 1.790 a 1.228 municipios, pues en muchos de ellos no se dispone de los datos relativos al período analizado. 
CUADRO 1

Estimaciones del modelo global (PIB per cápita)

\begin{tabular}{lcc}
\hline & $\begin{array}{c}\text { Número de } \\
\text { municipios }\end{array}$ & $\begin{array}{c}\text { Efecto del } \\
\text { FNE }\end{array}$ \\
\hline $\begin{array}{l}\text { Resultado sin controles }\left(X_{i}\right) \\
\text { (Modelo 1) }\end{array}$ & 1790 & $0,082^{*}$ \\
$\begin{array}{l}\text { Resultado con controles }\left(X_{i}\right) \\
\text { (Modelo 2) }\end{array}$ & 1228 & $0,085^{*}$ \\
\hline
\end{tabular}

Fuente: elaboración propia.

* Significativos al $5 \%$.

FNE: Fondo Constitucional de Financiamiento del Nordeste. PIB: producto interno bruto.

CUADRO 2

\section{Estimaciones del modelo con efecto de umbral \\ (PIB per cápita)}

\begin{tabular}{lcc}
\hline Grupo (intervalo para $\left.y_{i},{ }_{0}\right)$ & $\begin{array}{c}\text { Número de } \\
\text { municipios }\end{array}$ & $\begin{array}{c}\text { Efecto del } \\
\text { FNE }\end{array}$ \\
\hline $\begin{array}{l}\text { Resultado sin controles }\left(X_{i}\right) \text { (Modelo 3) } \\
\text { Inferior a } 4105 \text { reales }\end{array}$ & 1493 & $0,067^{*}$ \\
Superior a 4105 reales & 297 & $0,117^{*}$ \\
Resultado con controles $\left(X_{i}\right)$ (Modelo 4) & & \\
Inferior a 2 143 reales & 204 & $-0,007$ \\
Entre 2 143 y 3 866 reales & 794 & $0,078^{*}$ \\
Entre 3 866 y 7406 reales & 177 & $0,109^{* *}$ \\
Superior a 7406 reales & 55 & 0,173 \\
\hline
\end{tabular}

Fuente: elaboración propia.

* Significativos al 5\%; ** significativos al $10 \%$. FNE: Fondo Constitucional de Financiamiento del Nordeste. PIB: producto interno bruto.

Es interesante observar que en estos modelos tradicionales el efecto del FNE fue positivo y significativo, con y sin los controles relacionados con los cofactores determinantes del crecimiento económico, un resultado cualitativo diferente del observado en Resende (2012a).

Sin embargo, las pruebas del multiplicador de Lagrange indicaron un rechazo de los modelos globales en favor de los modelos con efecto de umbral con dos grupos, sin los controles $\left(X_{i}\right)$, y con cuatro grupos, incluyéndolos. En el primer caso, se obtiene un grupo formado por municipios con un PIB per cápita inicial menor de 4.105 reales (1.493 municipios) y otro con un PIB per cápita inicial superior a ese valor (297 municipios). Los efectos estadísticamente significativos del FNE en el crecimiento son 0,067 con respecto al grupo de menores ingresos y 0,117 con relación al de mayores ingresos. Esto significa que el volumen de recursos per cápita del FNE produce un efecto mayor en el crecimiento del ingreso de los municipios más desarrollados desde el punto de vista económico. En los municipios del primer grupo, cada incremento del $10 \%$ en los financiamientos per cápita del FNE se traduce en un aumento de 0,67 puntos porcentuales en la tasa media de crecimiento del PIB per cápita, mientras que en los municipios del segundo grupo ese incremento es de 1,17 puntos porcentuales.

Cabe señalar que conforme con el modelo global sin controles (véase el cuadro 1), ese aumento sería de 0,82 puntos porcentuales en todos los municipios. En contraste, una de las ventajas del modelo con efecto de umbral consiste en la posibilidad de establecer diferencias en los efectos del FNE e identificar la variable que determina esa diferenciación. Los resultados del modelo con efecto de umbral sin control de este estudio sugieren que la influencia de los recursos del FNE en el crecimiento económico es más intensa en los municipios con ingresos per cápita más altos. Esto podría obedecer al mayor dinamismo de su actividad económica y a la mayor madurez de sus empresarios.

Al controlar otros cofactores de crecimiento, el primer grupo resulta compuesto por municipios con un PIB per cápita inicial inferior a 2.143 reales (204 municipios), el segundo por municipios con un PIB per cápita inicial superior o igual a 2.143 reales e inferior a 3.866 reales (794 municipios), el tercero por aquellos con un PIB per cápita inicial mayor o igual a 3.866 reales e inferior a 7.406 reales (177 municipios) y el cuarto por aquellos con un valor mayor o igual a 7.406 reales (55 municipios).

Salvo en los grupos intermedios (el segundo y el tercero), la estimación de los efectos del FNE en el crecimiento del PIB per cápita no es estadísticamente significativa. Vale la pena señalar que los efectos medios por grupos se captan independientemente del volumen agregado recibido por ellos. En el cuadro 3 se denota, por ejemplo, que los grupos donde los efectos del FNE no fueron estadísticamente significativos son los que recibieron menores y mayores volúmenes de recursos per cápita.

CUADRO 3

Volumen del FNE por clubes de crecimiento, 2002-2006

\begin{tabular}{lrc}
\hline $\begin{array}{l}\text { Clubes } \\
\text { (PIB per cápita) }\end{array}$ & $\begin{array}{c}\text { Volumen FNE } \\
\text { (reales de 2007) }\end{array}$ & $\begin{array}{c}\text { Volumen per cápita } \\
\text { medio FNE } \\
\text { (reales de 2007) }\end{array}$ \\
\hline 2143 reales & 452397355,43 & 199,91 \\
$2143-3$ 866 reales & 3281973904,51 & 254,02 \\
3 866-7 406 reales & 2364298679,18 & 314,32 \\
7 406 reales & 2700014000,88 & 1716,11 \\
Total & 8798683940,00 & 319,20 \\
\hline
\end{tabular}

Fuente: elaboración propia.

FNE: Fondo Constitucional de Financiamiento del Nordeste. PIB: producto interno bruto. 
En los grupos de municipios donde el efecto del FNE fue significativo, el cuadro general es bastante similar al caso en que no se usan controles en el modelo de regresión. En el segundo grupo, cada incremento del $10 \%$ en los financiamientos per cápita del FNE conduce a un aumento de 0,78 puntos porcentuales en la tasa media de crecimiento del PIB per cápita de los municipios, mientras que en el tercero el aumento es de 1,09 puntos porcentuales. Esto confirma la evidencia de que los financiamientos del FNE producen un efecto creciente de acuerdo con el nivel de ingreso inicial de los municipios.

Otra constatación importante es que los efectos del FNE se advierten en la gran mayoría de los municipios de referencia. Se trata de aquellos que componen el segundo y tercer grupos, que representan aproximadamente el $79 \%$ de la muestra. La distinción endógena de municipios que registran o no efectos significativos del FNE permite un análisis de eficacia con respecto a los grupos de municipios clasificados según la PNDR (Ministerio de Integración Nacional, 2005).

Los cuatro grupos formados en este trabajo pueden denominarse clubes de crecimiento, pues los municipios que los componen presentan patrones de crecimiento del PIB per cápita similares, teniendo en cuenta la influencia conjunta de los otros cofactores del modelo. Así, es de esperar que el financiamiento del FNE produzca efectos diferenciados en municipios con diferentes niveles de PIB per cápita inicial. La comparación entre los municipios que componen los clubes definidos en este trabajo con los clasificados de acuerdo con la PNDR se detalla en el cuadro 4.

En el cuadro 4, las filas representan los clubes formados por el PIB per cápita inicial, mientras que las columnas se refieren a la clasificación de los municipios según la PNDR. Estos pueden clasificarse como de bajos ingresos, estancados, dinámicos y de altos ingresos, según la combinación tabular ad hoc del ingreso per cápita inicial y del crecimiento verificado en la década de $1990^{20}$. De los 379 municipios de la muestra clasificados como de bajos ingresos por la PNDR, 300 se encuentran en clubes de crecimiento que resultaron estadísticamente sensibles a los efectos del FNE (Grupo 2-270, y Grupo 3-30). De esta forma, si los municipios de bajos ingresos reciben el mismo volumen de financiamiento per cápita en forma lineal, la eficacia esperada es de alrededor del $79,1 \%$. Desde esa misma perspectiva, las estimaciones de eficacia en el caso de los municipios clasificados como estancados, dinámicos y de altos ingresos son del $83,4 \%$, el $75,7 \%$ y el $59,4 \%$, respectivamente.

La eficacia relativamente menor en los municipios de altos ingresos confirma la tendencia de la PNDR a dar prioridad (no exclusiva, cabe subrayar) a los municipios de las tres primeras categorías (de bajos ingresos, estancados y dinámicos), visto que el retorno del fondo sería mayor en esos municipios. Por otra parte, la constatación de efectos diferenciados entre los municipios con distintos niveles económicos revela la necesidad de un seguimiento más cuidadoso de los municipios que no responden adecuadamente a los flujos de inversiones.

Debido a que los grupos incluyen municipios con bajos y altos ingresos per cápita, se debe prestar atención a

20 Corresponde señalar que también existe una diferencia con respecto al año de referencia de la clasificación de la PNDR según el ingreso per cápita inicial y al PIB per cápita inicial de los clubes de crecimiento formados.

CUADRO 4

Clasificación de los municipios. Clubes de crecimiento según la PNDR

\begin{tabular}{|c|c|c|c|c|c|}
\hline \multirow{2}{*}{ Clubes según intervalos del РІВ } & \multicolumn{4}{|c|}{ Política Nacional de Desarrollo Regional (PNDR) } & \multirow{2}{*}{ Total } \\
\hline & Bajos ingresos & Estancados & Dinámicos & Altos ingresos & \\
\hline 2143 reales 1$)$ & $\begin{array}{r}74(36,3) \\
(19,5)\end{array}$ & $\begin{array}{r}54(26,5) \\
(13,2)\end{array}$ & $\begin{array}{r}76(37,3) \\
(18,6)\end{array}$ & $\begin{array}{r}0(0) \\
(0)\end{array}$ & 204 \\
\hline 2 143-3 866 reales 2) & $\begin{array}{r}270(34,1) \\
(71,2)\end{array}$ & $\begin{array}{r}262(33,1) \\
(64,1)\end{array}$ & $\begin{array}{r}253(31,9) \\
(62,0)\end{array}$ & $\begin{array}{r}7(0,9) \\
(21,9)\end{array}$ & 792 \\
\hline $3866-7406$ reales 3) & $\begin{array}{r}30(16,9) \\
(7,9)\end{array}$ & $\begin{array}{r}79(44,6) \\
(19,3)\end{array}$ & $\begin{array}{r}56(31,6) \\
(13,7)\end{array}$ & $\begin{array}{r}12(6,8) \\
(37,5)\end{array}$ & 177 \\
\hline 7406 reales 4$)$ & $\begin{array}{r}5(9,1) \\
(1,3)\end{array}$ & $\begin{array}{r}14(25,5) \\
(3,4)\end{array}$ & $\begin{array}{r}23(41,8) \\
(5,6)\end{array}$ & $\begin{array}{r}13(23,6) \\
(40,6)\end{array}$ & 55 \\
\hline Total municipios & 379 & 409 & 408 & 32 & 1228 \\
\hline
\end{tabular}

Fuente: elaboración propia.

1) PIB per cápita inferior a 2.143 reales.

2) PIB per cápita superior o igual a 2.143 reales e inferior a 3.866 reales.

3) PIB per cápita superior o igual a 3.866 reales e inferior a 7.406 reales.

4) PIB per cápita superior o igual a 7.406 reales. 
los factores locales específicos que dificultan el crecimiento inducido por las inversiones. Entre los factores de la oferta, la adecuación del mercadeo y el seguimiento de los proyectos son fundamentales para que estos tengan las repercusiones esperadas. En ese sentido, cabe señalar que una parte de los recursos del FNE destinados a los agricultores familiares (PRONAF) pasó recientemente a estar bajo el seguimiento sistematizado de agentes de crédito en programas de microfinanciamientos (programa AgroAmigo). Esto puede ser un factor alentador, sobre todo para los municipios de bajos ingresos con una mayor participación del sector agrícola. Debido a que la consolidación de este programa es posterior al período investigado en este trabajo, se recomienda efectuar una actualización temporal de las dinámicas de crecimiento en el Nordeste en futuras investigaciones.

Con respecto a la demanda, también es importante la organización estructurada de los agentes económicos locales para que puedan desarrollar y aplicar sus habilidades empresariales de forma coordinada. Con tal fin se debe promover la interacción entre el gobierno local, las agencias financiadoras y las instituciones de apoyo técnico y científico, de modo que la inversión se convierta en un factor de producción y productividad sostenidas en lugar de un mecanismo de reproducción del contexto económico actual.

\section{VI}

\section{Consideraciones finales}

En este artículo se procuró verificar los efectos del Fondo Constitucional de Financiamiento del Nordeste en el crecimiento económico de los municipios nordestinos durante la década de 2000. Para ello se utilizó un marco empírico basado en modelos de crecimiento (Barro y Sala-i-Martin, 1991) que permiten la formación de clubes de convergencia y heterogeneidad en los efectos de los cofactores de acuerdo con el nivel de desarrollo inicial del municipio (Durlauf y Johnson, 1995).

Una de las ventajas de ese modelo no lineal consiste en poder determinar, mediante un proceso de selección endógena, la composición de los "clubes" de municipios que presentan patrones de crecimiento similares entre sí y diferentes (estadísticamente) entre los grupos. Al probar esa posibilidad con respecto al crecimiento del PIB per cápita en los municipios del Nordeste en el período 2002-2008, se constató la existencia de cuatro clubes de convergencia, con efectos diferenciados del FNE, discriminados según el nivel del PIB per cápita inicial de los municipios.

Los efectos del FNE no resultaron estadísticamente significativos en los municipios con un PIB per cápita de hasta 2.143 reales (204 municipios) y en aquellos con valores superiores a 7.406 reales (55 municipios). En cambio, se registraron efectos positivos y estadísticamente significativos en los municipios con PIB per cápita intermedios, con valores comprendidos entre 2.143 reales y 3.866 reales (792 municipios) y entre 3.866 reales y 7.406 reales (177 municipios). En el primer grupo, un incremento del $10 \%$ en el volumen de recursos del
FNE per cápita se traduciría en un aumento medio de 0,78 puntos porcentuales en la tasa de crecimiento del $\mathrm{PIB}$, mientras que en el segundo grupo el incremento sería de 1,09 puntos porcentuales. Se trata de efectos considerables, vistos los antecedentes de las tasas de crecimiento de la región.

En conjunto, los resultados indican que el FNE tuvo un efecto medio positivo en el crecimiento de los municipios del Nordeste en el período de 2002 a 2008. Si bien el efecto positivo general es importante para sancionar este instrumento de política regional, es necesario tener en cuenta las advertencias metodológicas y las características específicas de los resultados.

Conviene destacar que, en la definición de la ecuación econométrica para estimar los efectos del FNE en los municipios, se procuró seguir los patrones de la literatura empírica de crecimiento económico que se basan en modelos para datos de corte transversal. $\mathrm{Si}$ bien una evaluación mediante datos de panel podría ser más rica en virtud de la multidimensionalidad de los datos, la limitada disponibilidad de información impidió programar esa evaluación.

Otra restricción, también relacionada con la disponibilidad de datos, es la ausencia de la variable explicativa "capital físico", generalmente presente en estudios de este tipo. Se espera que la variable "hogares con agua por cañería", sustitutiva del capital físico municipal, capte parcialmente la variación y reduzca en gran medida la posibilidad de sesgos en las estimaciones. Sería recomendable realizar pruebas de 
especificación para investigar más a fondo ese problema, pero al tratarse de modelos con efecto de umbral esas técnicas todavía no han sido propuestas. Por último, si bien es posible incluir más de una variable de umbral en la formación de grupos de municipios en el proceso de estimación (como ocurre en algunos trabajos que emplean modelos con ese tipo de no linealidad), ello aumentaría la complejidad del análisis en el sentido de identificar el factor que determina la formación del grupo de convergencia. El uso de la variable ingreso per cápita inicial como única variable de umbral en los modelos de crecimiento económico es prácticamente universal.

En futuros trabajos complementarios se podrían estimar modelos de crecimiento relacionados con otros niveles de agregación regional (por ejemplo, microrregiones), a objeto de captar algún efecto de escala regional o disminuir posibles problemas de medición de variables en el sector municipal ${ }^{21}$.

Con respecto a los resultados específicos del trabajo, la ausencia de efectos significativos en el crecimiento de los municipios con menos ingresos subraya la necesidad de mejorar el seguimiento de la cantidad y la calidad de los recursos que les son destinados, como también de los factores locales que representan obstáculos para el crecimiento económico. El mismo razonamiento se aplica también a los municipios con mayores ingresos.

\footnotetext{
${ }^{21}$ Este posible problema se aborda en Resende (2012a).
}

\section{ANEXO}

CUADRO A.1

\section{Variables explicativas}

\begin{tabular}{|c|c|c|c|c|}
\hline Variable & Definición & Fuente & Media & $\begin{array}{l}\text { Desviación } \\
\text { estándar }\end{array}$ \\
\hline$\overline{F_{i}}$ & $\begin{array}{l}\text { Media del total de financiamientos del FNE per cápita en el período } \\
\text { 2002-2006 (en reales de 2007) }\end{array}$ & BNB & 64,01 & 234,99 \\
\hline$y_{i 0}$ & PIB per cápita municipal en 2002 (en reales de 2007) & Ipeadata & 3712,06 & 7395,23 \\
\hline$(n+\delta+d)$ & $\begin{array}{l}\text { Tasa media de crecimiento poblacional entre } 2002 \text { y } 2008 \text {, } \\
\text { más } 0,05 \text { de crecimiento tecnológico y depreciación }\end{array}$ & Ipeadata & 0,0572 & 0,01 \\
\hline$e d u$ & Media de años de estudio de personas con 25 años & Ipeadata & 2,90 & 0,83 \\
\hline infra & Proporción de hogares con agua por cañería (en porcentajes) & Ipeadata & 42,95 & 19,47 \\
\hline gov & Media de la razón entre gasto corriente y PIB municipal en 2002 & Ipeadata & 45,59 & 19,32 \\
\hline dist & Distancia del municipio de la capital del respectivo estado & Ipeadata & 226,58 & 148,89 \\
\hline opc & $\begin{array}{l}\text { Total de operaciones de crédito per cápita en } 2002 \text { (en reales } \\
\text { de 2007) }\end{array}$ & $\begin{array}{l}\text { Banco Central } \\
\text { del Brasil }\end{array}$ & 436,86 & 4258,79 \\
\hline semi & Variable igual a 1 si está situado en el semiárido, 0 de otra manera & Ipeadata & 0,63 & 0,48 \\
\hline
\end{tabular}

Fuente: elaboración propia.

FNE: Fondo Constitucional de Financiamiento del Nordeste.

PIB: producto interno bruto.

BNB: Banco do Nordeste do Brasil. 
CUADRO A. 2

Estimaciones de los modelos global y con efecto de umbral sin las variables de control

(PIB per cápita)

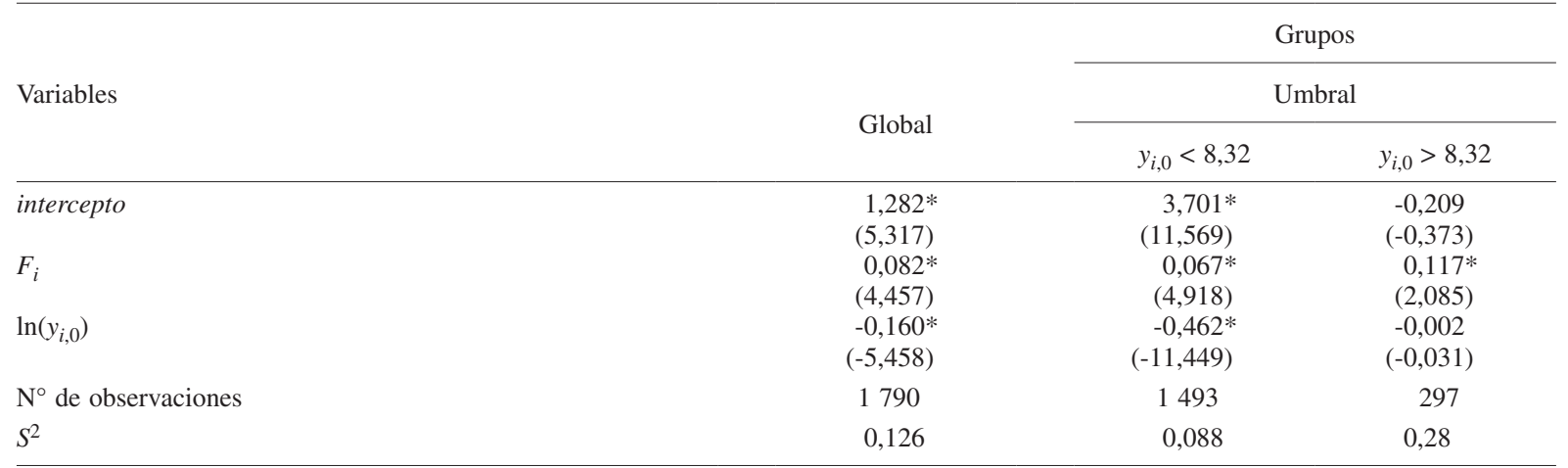

Fuente: elaboración propia.

Valor $t$ entre paréntesis.

$S^{2}$ : varianza muestral del modelo.

* Significativo al 5\%.

PIB: producto interno bruto.

CUADRO A.3

Estimaciones de los modelos global y de efecto de umbral mediante variables de control

(PIB per cápita)

\begin{tabular}{|c|c|c|c|c|c|}
\hline \multirow{2}{*}{ Variables } & \multirow[b]{2}{*}{ Global } & \multicolumn{4}{|c|}{ Grupos } \\
\hline & & $y_{i, 0}<7,67$ & $7,67 \leq y_{i, 0}<8,26$ & $8,26 \leq y_{i, 0}<8,91$ & $y_{i, 0}>8,91$ \\
\hline \multirow[t]{2}{*}{ intercepto } & $0,896 *$ & $-0,199$ & $3,368 *$ & 2,479 & 3,018 \\
\hline & $(2,282)$ & $(-0,170)$ & $(7,565)$ & $(0,974)$ & $(0,876)$ \\
\hline \multirow[t]{2}{*}{$F_{i}$} & $0,085^{*}$ & $-0,007$ & $0,078 *$ & $0,109 * *$ & 0,173 \\
\hline & $(3,242)$ & $(-0,441)$ & $(4,606)$ & $(1,958)$ & $(1,588)$ \\
\hline \multirow[t]{2}{*}{$\ln \left(y_{i, 0}\right)$} & $-0,088 *$ & 0,068 & $-0,419 *$ & $-0,421 * *$ & $-0,5668$ \\
\hline & $(-2,053)$ & $(0,402)$ & $(-8,334)$ & $(-1,665)$ & $(-2,200)$ \\
\hline \multirow[t]{2}{*}{$(n+\delta+d)_{i}$} & $-0,127 *$ & $-0,157 *$ & $-0,120 *$ & $-0,377^{*}$ & 1,496 \\
\hline & $(-2,876)$ & $(-3,746)$ & $(-2,683)$ & $(-2,920)$ & $(1,493)$ \\
\hline \multirow[t]{2}{*}{$e d u_{i}$} & 0,044 & 0,002 & $0,148 *$ & 0,091 & 0,398 \\
\hline & $(1,122)$ & $(0,030)$ & $(3,408)$ & $(0,688)$ & $(0,997)$ \\
\hline \multirow[t]{2}{*}{ infra $_{i}$} & $-0,029 *$ & $-0,023 * *$ & $-0,031 *$ & 0,121 & $-0,052$ \\
\hline & $(-2,313)$ & $(-1,748)$ & $(-2,213)$ & $(1,556)$ & $(-0,208)$ \\
\hline \multirow[t]{2}{*}{$\operatorname{gov}_{i}$} & $-0,089 *$ & $-0,039$ & $-0,073^{*}$ & $-0,137$ & $0,838^{*}$ \\
\hline & $(-2,265)$ & $(-0,819)$ & $(-2,245)$ & $(-1,479)$ & $(2,009)$ \\
\hline \multirow[t]{2}{*}{ dist $_{i}$} & 0,006 & 0,005 & $-0,001$ & 0,032 & 0,048 \\
\hline & $(0,015)$ & $(1,079)$ & $(-0,101)$ & $(1,062)$ & $(0,566)$ \\
\hline \multirow[t]{2}{*}{$o p c_{i}$} & 0,004 & 0,005 & $0,006^{* *}$ & $-0,005$ & 0,044 \\
\hline & $(0,909)$ & $(1,080)$ & $(1,666)$ & $(-0,523])$ & $(0,698)$ \\
\hline \multirow[t]{2}{*}{$\operatorname{semi}_{i}$} & $-0,077 *$ & 0,010 & $-0,071 *$ & $-0,141$ & $-0,290^{*}$ \\
\hline & $(-3,089)$ & $(0,310)$ & $(-2,634)$ & $(-1,386)$ & $(3,018)$ \\
\hline $\mathrm{N}^{\circ}$ de observaciones & 1228 & 204 & 792 & 177 & 55 \\
\hline$S^{2}$ & 0,107 & 0,046 & 0,057 & 0,129 & 0,806 \\
\hline
\end{tabular}

Fuente: elaboración propia.

Valor $t$ entre paréntesis.

$S^{2}$ : varianza muestral del modelo.

* Significativo al 5\%; ** significativo al $10 \%$.

PIB: producto interno bruto. 


\section{Bibliografía}

Aghion, P., P. Howitt y D. Mayer-Foulkes (2004), "The effect of financial development on convergence: theory and evidence", NBER Working Paper, $\mathrm{N}^{\circ}$ 10358, Cambridge, Massachusetts, National Bureau of Economic Research.

Azariadis, C. y A. Drazen (1990), "Threshold externalities in economic development", The Quarterly Journal of Economics, vol. 105, $\mathrm{N}^{\circ}$ 2, Oxford, Oxford University Press.

Banco do Nordeste (2011), Fundo Constitucional de Financiamento do Nordeste - FNE. Relatório de resultados e impactos: Exercício de 2011 - Primeiro semestre.

Barro, R. y X. Sala-i-Martin (1992), "Convergence", Journal of Political Economy, vol. 100, $\mathrm{N}^{\circ} 2$, Chicago, University of Chicago Press.

(1991), "Convergence across states and regions", Brookings Papers on Economic Activity, vol. 22, N ${ }^{\circ}$, Washington, D.C., The Brookings Institution.

Baumol, J.W. (1986), "Productivity growth, convergence, and welfare: what the long-run data show", The American Economic Review, vol. 76, $\mathrm{N}^{\circ}$ 5, Nashville, Tennessee, American Economic Association, diciembre.

Bayoumi, T. y O. Melander (2008), "Credit matters: empirical evidence on U.S. macro-financial linkages", IMF Working Paper, $\mathrm{N}^{\circ}$ 08/169, Washington, D.C., Fondo Monetario Internacional.

Bencivenga, V.R. y B.D. Smith (1991), "Financial intermediation and endogenous growth", Review of Economic Studies, vol. 58, $\mathrm{N}^{\circ} 2$, Oxford, Oxford University Press.

Bourguignon, F. (2002), "The distributional effects of growth: case studies vs. cross-country regressions", DELTA Working Paper, $\mathrm{N}^{\circ}$ 2002-23, París, DELTA (École normale supérieure).

Boyd, J.H. y E.C. Prescott (1986), "Financial intermediarycoalitions", Journal of Economic Theory, vol. 38, $\mathrm{N}^{\circ} 2$, Amsterdam, Elsevier.

Brasil (1999), "Lei no 9.808, de 20 de julho de 1999. Define diretrizes e incentivos fiscais para o desenvolvimento regional e dá outras providências" [en línea] http://www.planalto.gov.br.

Cabral, E.C.S. (2008), "Convergência de renda per capita entre os Estados brasileiros de 1939 a 2004", tesis (Doctorado en Economía), Brasilia, Universidad de Brasilia.

Chinelatto Neto, A. (2007), "Relações entre crédito e crescimento econômico no Brasil, 2000 a 2006", tesis (Doctorado en Economía), Viçosa, Minas Gerais, Universidad Federal de Viçosa.

Dall'Erba, S., R. Guillain y J.L. Gallo (2011), “Impact of structural funds on regional growth: how to reconsider a 7 year-old black-box", Documento de trabajo.

Dall'Erba, S. y H.L.F. de Groot (2006), "A meta-analysis of EU regional policy evaluation", documento presentado en la $53 \mathrm{rd}$ Annual North American Meetings of the RSAI, Toronto.

De Gregorio, J. y P.E. Guidotti (1995), "Financial development and economic growth", World Development, vol. 23, $\mathrm{N}^{\circ} 3$, Amsterdam, Elsevier.

Durlauf, S.N. y P.A. Johnson (1995), "Multiple regimes and crosscountry growth behavior", Journal of Applied Econometrics, vol. 10, $\mathrm{N}^{\circ} 4$, John Wiley \& Sons, Ltd.

Durlauf, S.N., P.A. Johnson y J.R.W. Temple (2004), "Growth econometrics", Vassar College Department of Economics Working Paper, $\mathrm{N}^{\circ} 61$.

Durlauf, S.N. y D.T. Quah (1999), "The new empirics of economic growth", Handbook of Macroeconomics, J.B. Taylor y M. Woodford (ed.), vol. 1, Amsterdam, Elsevier.

Goldsmith, R.W. (1969), Financial Structure and Development, New Haven, Connecticut, Yale University Press.

Greenwood, J. y B. Jovanovic (1990), "Financial development, growth and the distribution of income", Journal of Political Economy, vol. 98, $\mathrm{N}^{\circ} 5$, Chicago, University of Chicago Press.
Hansen, B.E. (2000), "Sample splitting and threshold estimation", Econometrica, vol. 68, $\mathrm{N}^{\circ} 3$, Nueva York, The Econometric Society.

(1996), "Inference when a nuisance parameter is not identiffed under the null hypothesis", Econometrica, vol. 64, $\mathrm{N}^{\circ} 2$, Nueva York, The Econometric Society.

Jappelli, T. y M. Pagano (1994), "Saving, growth and liquidity constraints", Quarterly Journal of Economics, vol. 109, № 1 , Cambridge, Massachusetts, The MIт Press.

Keynes, J.M. (1943), Teoría general de la ocupación, el interés y el dinero, México, D.F., Fondo de Cultura Económica. Publicado originalmente en inglés en 1936.

King, R.G. y R. Levine (1993), "Finance, entrepreneurship, and growth", Journal of Monetary Economics, vol. 32, $\mathrm{N}^{\circ} 3$, Amsterdam, Elsevier.

Levine, R. (2004), "Finance and growth: theory and evidence", NBER Working Paper, $\mathrm{N}^{\circ}$ 10766, Cambridge, Massachusetts, National Bureau of Economic Research.

(1992), "Financial structure and economic development", Policy Research Working Paper Series, N ${ }^{\circ} 449$, Washington, D.C., Banco Mundial.

Mankiw, N., G.D. Romer y D.N. Weil (1992), "A contribution to the empirics of economics", Quarterly Journal of Economics, vol. 107, $\mathrm{N}^{\circ} 2$, Oxford, Oxford University Press.

Marques Jr., T.E. y S.S. Porto Jr. (2004), "Desenvolvimento financeiro e crescimento econômico no Brasil - Uma avaliação econométrica", Trabalho para Discussão, $\mathrm{N}^{\circ} 11$, Programa de Posgrado en Economía/Universidad Federal de Rio Grande do Sul (UFRGS).

Matos, O.C. (2002), "Desenvolvimento do sistema financeiro e crescimento econômico no Brasil: Evidência de causalidade", Trabalho para discussão, $\mathrm{N}^{\circ} 49$, Banco Central del Brasil.

McKinnon, R. (1973), Money and Capital in Economic Development, Washington, D.C., The Brookings Institute.

Mesquita, A.M. (1996), "Avaliação econômica do Fundo Constitucional de Financiamento do Nordeste (FNE)", Dissertação de Mestrado, Minas Gerais, Universidad Federal de Minas Gerais.

Ministerio de Integración Nacional (2010), 20 Anos de Fundos Constitucionais de Financiamento (FCO-FNE-FNO), Brasilia.

(2005), Plano Nacional de Desenvolvimento Regional, Brasilia.

Oliveira, H.C. de y E.P. Domingues (2005), "Considerações sobre o impacto dos Fundos Constitucionais de Financiamento do Norte e do Centro-Oeste na redução da desigualdade regional no Brasil", Anais do XXXIII Encontro Nacional de Economia, Río de Janeiro, Asociación Nacional de Centros de Posgrado en Economía (ANPEC).

Oliveira, J.L. y otros (2011), "Dinâmica regional e convergência de renda: um estudo para os municípios cearenses no período recente", Dinâmica regional e convergência de renda. Uma análise para os municípios brasileiros selecionados no período 2002-2007, Brasilia, Instituto de Investigación Económica Aplicada (IPEA).

Penna, C.M. (2011), "Convergência dos PIBs estaduais per capita no Brasil", tesis de doctorado, Fortaleza, CAEN.

Phillips, P.C.B. y D. Sul (2007), "Transition modeling and econometric convergence tests", Econometrica, vol. 75, $\mathrm{N}^{\circ} 6$, Nueva York, The Econometric Society.

Resende, G.M. (2012a), "Measuring micro- and macro-impacts of regional development policies: the case of the Northeast Regional Fund (FNE) industrial loans in Brazil, 2000-2006", Regional Studies, vol. 48, $\mathrm{N}^{\circ} 4$, Taylor \& Francis.

(2012b), "Micro e macroimpactos de políticas de desenvolvimento regional: o caso dos empréstimos do FNEindustrial no estado do Ceará", Texto para Discussão, N ${ }^{\circ} 1777$, Río de Janeiro, Instituto de Investigación Económica Aplicada (IPEA). 
Rocha, B.P. y M.I. Nakane (2007), "Sistema financeiro e desenvolvimento econômico: evidências de causalidade em um painel para o Brasil", Anais do XXXV Encontro Nacional de Economia, Río de Janeiro, Asociación Nacional de Centros de Posgrado en Economía (ANPEC).

Rodrigues, M.T. (1998), "Eficiência alocativa do Fundo Constitucional de Financiamento do Nordeste (FNE): uma visão de insumoproduto", Dissertação, São Paulo, Escuela Superior de Agricultura Luiz de Queiroz/Universidad de São Paulo.

Schumpeter, J.A. (1934), The Theory of Economic Development, Cambridge, Massachusetts, Harvard University Press.

Shaw, E.S. (1973), Financial Deepening in Economic Development, Nueva York, Oxford University Press.
Silva, A.M., G.M. Resende y R. Silveira Neto (2009), "Eficácia do gasto público: uma avaliação do FNE, FNO e FCO", Estudos econômicos, vol. 39, $\mathrm{N}^{\circ} 1$, São Paulo.

Soares, R., J. Sousa y A. Pereira Neto (2009), “Avaliação de impacto do FNE no emprego, na massa salarial e no salário médio em empreendimentos financiados", Revista Econômica do Nordeste, vol. 40, $\mathrm{N}^{\circ}$ 1, Fortaleza, Banco do Nordeste.

Trompieri Neto, N., I. Castelar y F.C. Linhares (2008), "Convergência de renda dos estados brasileiros: uma abordagem de painel dinâmico com efeito threshold", Anais do XXXVI Encontro Nacional de Economia, Río de Janeiro, Asociación Nacional de Centros de Posgrado en Economía (ANPEC).

Tsangarides, C.G. (2005), "Growth empirics under model uncertainty: is Africa different?, IMF Working Papers, $\mathrm{N}^{\circ} 05 / 18$, Washington, D.C., Fondo Monetario Internacional. 